\title{
Dark Matter in the Coming Decade: Complementary Paths to Discovery and Beyond
}

\begin{abstract}
Daniel Bauer, Fermilab; James Buckley, Washington University; Matthew Cahill-Rowley, SLAC; Randel Cotta, University of California, Irvine; Alex Drlica-Wagner, SLAC; Jonathan L. Feng*, University of California, Irvine; Stefan Funk, SLAC; JoAnne Hewett, SLAC; Dan Hooper, Fermilab; Ahmed Ismail, SLAC; Manoj Kaplinghat*, University of California, Irvine; Alexander Kusenko, University of California, Los Angeles; Konstantin Matchev*, University of Florida; Daniel McKinsey, Yale University; Tom Rizzo, SLAC; William Shepherd, University of California, Santa Cruz; Tim M. P. Tait*, University of California, Irvine; Alexander M. Wijangco, University of California, Irvine; Matthew Wood, SLAC on behalf of the Snowmass 2013 Cosmic Frontier Working Groups 1-4
\end{abstract}

(Dated: 19 February 2015)

\section{INTRODUCTION}

Dark matter is five times as prevalent as normal matter in the Universe, but its identity is unknown. Its mere existence implies that our inventory of the basic building blocks of nature is incomplete, and uncertainty about its properties clouds attempts to fully understand how the Universe evolved to its present state and how it will evolve in the future. Dark matter is therefore a grand challenge for both fundamental physics and astronomy. At the same time, groundbreaking experiments are set to transform the field of dark matter in the coming decade. This prospect has drawn many new researchers to the field, which is now characterized by an extraordinary diversity of approaches unified by the common goal of discovering the identity of dark matter.

As we will discuss, a compelling solution to the dark matter problem requires synergistic progress along many lines of inquiry. Our primary conclusion is that the diversity of possible dark matter candidates requires a balanced program based on four pillars: direct detection experiments that look for dark matter interacting in the lab, indirect detection experiments that connect lab signals to dark matter in our own and other galaxies, collider experiments that elucidate the particle properties of dark matter, and astrophysical probes sensitive to non-gravitational interactions of dark matter such as dark matter densities in the centers of galaxies and cooling of stars.

In this Report we summarize ${ }^{1}$ the many dark matter searches currently being pursued in each of these four approaches. The essential features of broad classes of experiments are described, each with their own strengths and weaknesses. The goal of this Report is not to prioritize individual experiments, but rather to highlight the complementarity of the four general approaches that are required to sustain a vital dark matter research program. Complementarity also exists on many other levels, of course; in particular, complementarity within each approach is also important, but will be addressed by the Snowmass Cosmic Frontier subgroups that focus on each approach.

In Sec. II we briefly summarize what is known about dark matter and some of the leading particle candidates. In Sec. III, we discuss four broad categories of search strategies and summarize the current status of experiments in each area. We then turn to the complementarity of these approaches in Sec. IV. Conclusions are collected in Sec. V.

\footnotetext{
* Corresponding authors: jlf@uci.edu, mkapling@uci.edu, matchev@phys.ufl.edu, and ttait@uci.edu.

${ }^{1}$ Extended version of this report is available as [1.
} 


\section{EVIDENCE AND CANDIDATES}

Dark matter was first postulated in its modern form in the 1930s to explain the anomalously large velocities of galaxies in the Coma cluster [2]. Evidence for dark matter has grown steadily since then from data from galactic rotation curves [3 5], weak [6] and strong [7] lensing, hot gas in clusters [8, 9], the Bullet Cluster [10], Big Bang nucleosynthesis (BBN) [11], distant supernovae [12, 13, the statistical distribution of galaxies [14, 15] and the cosmic microwave background (CMB) [16, 17. Together, these data provide overwhelming evidence that the energy in dark matter is roughly a quarter of the total energy in the visible Universe and about five times the energy in normal matter.

All of this evidence for dark matter derives from its gravitational pull on visible matter. This does little to shed light on the identity of dark matter, since all particles interact universally through gravity. To make progress, dark matter must be detected through non-gravitational interactions. There are many possibilities. For reviews, see, e.g., Refs. [18 20].

In the case of weakly interacting massive particles (WIMPs), dark matter particles are produced in the hot early Universe and then annihilate in pairs. Those that survive to the present are known as "thermal relics" 21 24]. Such particles are generically predicted in models of physics beyond the standard model, including models with supersymmetry [25, 26] or extra spatial dimensions [27, 28]. Remarkably, if these particles interact through the weak interactions of the standard model, the resulting thermal relic density is $\Omega_{X} \sim \mathcal{O}(0.1)$, just right to be dark matter. This coincidence, the "WIMP miracle," provides strong motivation for dark matter with masses from $10 \mathrm{GeV}$ to $1 \mathrm{TeV}$ (or 10 to 1000 times the mass of the proton) and weak interactions with visible particles.

An alternative possibility is asymmetric dark matter [29 32]. In this case, there is a slight excess of dark particles over dark antiparticles in the early Universe. These annihilate until only the slight excess of dark particles remains. In many models, the dark matter asymmetry is related to the normal matter-antimatter asymmetry, and one expects the number of dark matter particles to be similar to the number of protons. Since dark matter contributes roughly five times more to the energy density of the Universe than normal matter, this scenario predicts dark matter particles with mass $\sim 1-10 \mathrm{GeV}$.

There are several other important dark matter candidates [33]. Axions [34 37] are strongly motivated by a severe problem of the standard model: the theory of the strong interactions naturally predicts large $\mathrm{CP}$ violating effects that have not been observed. Axions would resolve this problem elegantly by suppressing CP violation to experimentally allowed levels. Right-handed or sterile neutrinos are motivated by the observation of non-zero neutrino masses, and for certain ranges of masses and interaction strengths, they may be dark matter [38 40]. Alternatively, dark matter may be in a so-called hidden sector, which has its own set of matter particles and forces, through which the dark matter interacts with other currently unknown particles.

Although these dark matter candidates differ in important ways, in most cases, they have nongravitational interactions through which they may be detected. The non-gravitational interactions may be with any of the known particles or, as noted above for hidden sector dark matter, with other currently unknown particles. These possibilities are shown in Fig. 1, where the particles are grouped into four categories: nuclear matter; leptons; photons and other bosons; and other as-yet unknown particles. Dark matter may interact with one type of particle, or it may interact with several.

A complete research program in dark matter therefore requires a diverse set of experiments that together probe all possible types of couplings. At present, the experiments may be grouped into the following four categories:

- Direct Detection. Dark matter scatters off a detector, producing a detectable signal. Prime 


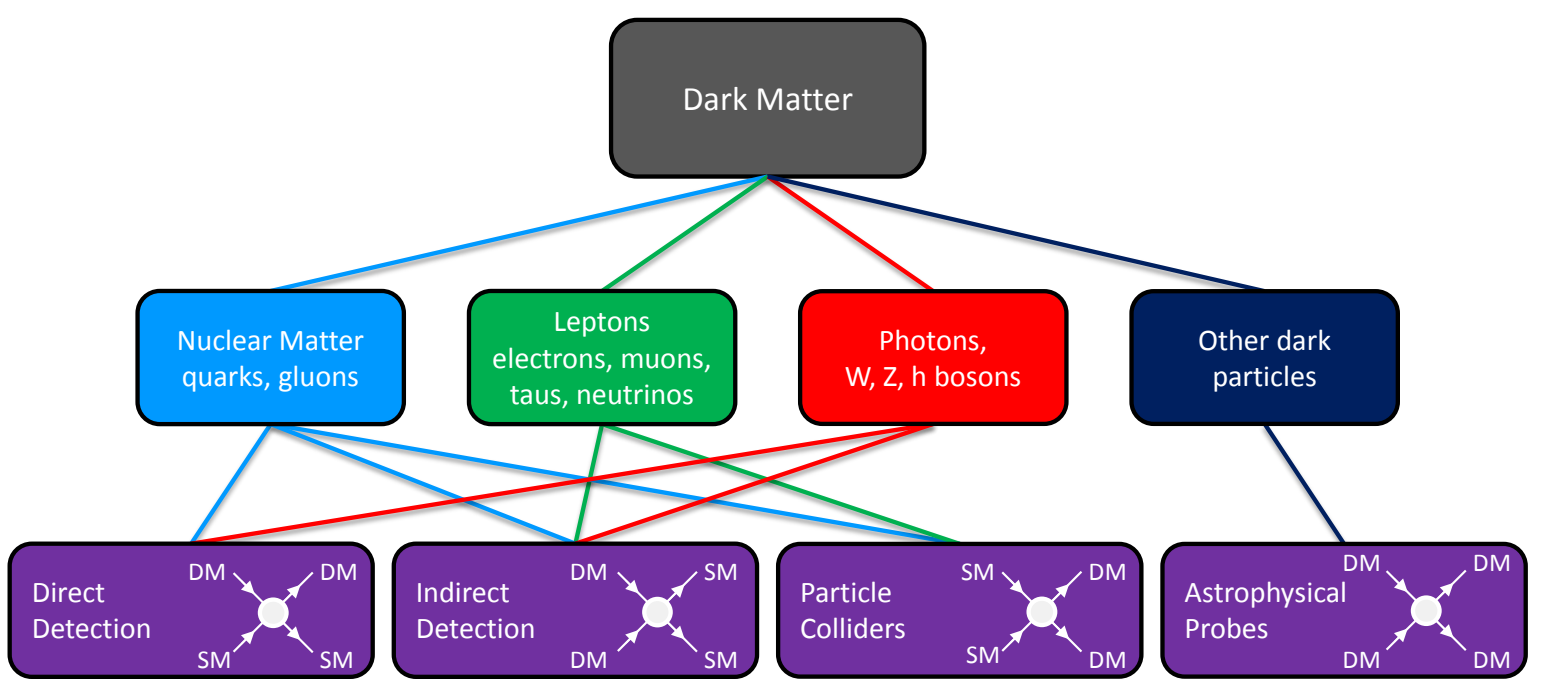

FIG. 1: Dark matter may have non-gravitational interactions with one or more of four categories of particles: nuclear matter, leptons, photons and other bosons, and other dark particles. These interactions may then be probed by four complementary approaches: direct detection, indirect detection, particle colliders, and astrophysical probes. The lines connect the experimental approaches with the categories of particles that they most stringently probe (additional lines can be drawn in specific model scenarios). The diagrams give example reactions of dark matter (DM) with standard model particles (SM) for each experimental approach.

examples are the detection of WIMPs through scattering off nuclei and the detection of axions through their interaction with photons in a magnetic field.

- Indirect Detection. Pairs of dark matter particles annihilate producing high-energy particles (antimatter, neutrinos, or photons). Alternatively, dark matter may be metastable, and its decay may produce the same high-energy particles.

- Particle Colliders. Particle colliders, such as the Large Hadron Collider (LHC) and proposed future lepton colliders, produce dark matter particles, which escape the detector, but are discovered as an excess of events with missing energy or momentum.

- Astrophysical Probes. The particle properties of dark matter are constrained through its impact on astrophysical observables. Examples include self-interaction of dark matter particles affecting central dark matter densities in galaxies (inferred from rotation velocity or velocity dispersion measures), mass of dark matter particle affecting dark matter substructure in galaxies (inferred from strong lensing data) and annihilation of dark matter in the early Universe affecting the Cosmic Microwave Background fluctuations.

These search strategies are shown in Fig. 1 and are connected to the particle interactions they most stringently probe. In the next Section, we briefly describe these four approaches and summarize their current status.

\section{THE FOUR PILLARS OF DARK MATTER DETECTION}

\section{A. Direct Detection}

Dark matter permeates the whole Universe, and its local density on Earth is known to be $5 \times 10^{-25} \mathrm{~g} / \mathrm{cm}^{3}$ to within a factor of 2 . This creates the opportunity to detect dark matter particles directly as they pass through and scatter off normal matter [41]. Such events are extremely 
rare, and so the direct detection approach requires sensitive detectors with exquisite background rejection [42. The expected signals depend on the nature of the dark matter particles and their interactions. For a list of current and planned experiments, see [3].

In the case of WIMPs, direct searches are extremely promising. Experimental techniques include detectors that record ionization, scintillation light, and phonons. The most sensitive of the detectors employ multiple techniques, and the interplay of each is used to discriminate against backgrounds. Depending on the target material, experiments can be sensitive to (a combination of) spin-dependent and spin-independent WIMP interactions with matter. The sensitivity of the current generation of detectors for spin-independent (spin-dependent) cross sections for scattering off protons is approaching $\sigma_{\mathrm{SI}}^{p} \sim 10^{-45} \mathrm{~cm}^{2}\left(\sigma_{\mathrm{SD}}^{p} \sim 10^{-39} \mathrm{~cm}^{2}\right)$ for WIMP masses of $\sim 100 \mathrm{GeV}$, with orders of magnitude improvement expected in the coming decade [42]. In the last years a number of experiments have reported potential signals that could be interpreted as very light ( 10 $\mathrm{GeV}) \mathrm{DM}$ particles. Although this interpretation is still inconclusive, it greatly motivates designing experiments with low threshold energies.

Axions also have strong prospects for direct detection. Cosmological and astrophysical constraints restrict the allowed axion mass range to be between $1 \mu \mathrm{eV}$ and $1 \mathrm{meV}$. In a static magnetic field, there is a small probability for cosmologically produced axions to be converted by virtual photons to real microwave photons by the Primakoff effect [44]. This would produce a monochromatic signal with a line width of $d E / E \sim 10^{-6}$, which could be detected in a high- $Q$ microwave cavity tunable over $\mathrm{GHz}$ frequencies. In the near future, these searches will be sensitive to models with axion mass $\sim \mu \mathrm{eV}$, which is the favored mass range if axions are a significant component of dark matter.

\section{B. Indirect Detection}

In contrast to direct detection experiments, indirect detection efforts do not aim to detect dark matter particles themselves. Instead, they attempt to detect the standard model particles that are produced in their annihilations or decays. Signals for indirect detection experiments include photons (gamma rays, X-rays, radio), neutrinos and cosmic rays (including positrons, electrons, antiprotons, and antideuterons). Many types of detectors and telescopes have been designed and deployed with these goals in mind, ranging from space- and ground-based gamma-ray telescopes and cosmic-ray detectors, to large underground, under-ice, and underwater neutrino telescopes [45. Current and planned indirect search experiments are listed in [46].

Motivating the existing and planned indirect detection efforts is the characteristic annihilation cross section of WIMP thermal relics. Although the precise value of this cross section depends on a number of model-dependent features, WIMP candidates that annihilate to the correct relic density to be dark matter typically have average cross sections (multiplied by the relative velocity of the annihilating WIMPs) of $\langle\sigma v\rangle \sim 3 \times 10^{-26} \mathrm{~cm}^{3} / \mathrm{s}$.

Excitingly, indirect detection experiments have started to reach the level of sensitivity required to discover WIMPs with this annihilation cross section (thermal WIMP). Current constraints from the Fermi Gamma-Ray Space Telescope observations of some Milky Way satellite galaxies have begun to exclude the low-mass region of some thermal WIMP models [47. On the other hand, there are excesses seen in gamma rays and microwaves towards the Galactic Center that are remarkably consistent with a thermal WIMP dark matter interpretation, though other explanations have not been ruled out.

Measurements of the cosmic-ray antiproton spectrum from PAMELA also constrain some of the thermal WIMP models, with further results expected from AMS-02 experiment on the International Space Station. Above about $10 \mathrm{GeV}$, there is an excess in the cosmic-ray positron flux which is 
seen by PAMELA, Fermi and the AMS-02 experiments. This has been interpreted as a sign of dark matter annihilating to leptons, although nearby pulsars could also provide an explanation. The indirect detection of dark matter is a major science goal of the kilometer-scale neutrino telescope IceCube as well. In contrast to other indirect searches, neutrino telescopes are most sensitive to WIMPs that annihilate in the core of the Sun. Current constraints from IceCube data have begun to exclude otherwise viable WIMP models.

Indirect searches are not limited to dark matter in the form of WIMPs. Sterile neutrinos, for example, are predicted to decay, leading to potentially observable X-ray spectral lines [48. Solar axion searches are sensitive to axions produced in the Sun (because of their coupling to photons) which then convert in the magnetic field of the detector [49].

\section{Particle Colliders}

Dark matter may also be produced in high-energy particle collisions. For example, if dark matter has substantial couplings to nuclear matter, it can be created in proton-proton collisions at the Large Hadron Collider (LHC). Once produced, dark matter particles will likely pass through detectors without a trace, but their existence may be inferred from an imbalance in the visible momentum, just as in the case of neutrinos. Searches for dark matter at the LHC are therefore typified by missing momentum, and can be categorized by the nature of the visible particles that accompany the dark matter production. Because backgrounds are typically smaller for larger values of missing momentum, collider searches tend to be most effective for low-mass dark matter particles, which are more easily produced with high momentum.

There are two primary mechanisms by which the LHC could hope to produce dark matter together with hadronic jets (see, e.g., Chapters 13 and 14 in Ref. [18]). In the first, two strongly interacting parent particles of the dark matter theory are produced, and each one subsequently decays into the dark matter and standard model particles, resulting in missing momentum plus two or more jets of hadrons. Since the production relies on the strong force, the rate of production is specified by the color charge, mass, and spin of the parent particles and is rather insensitive to the mass of the dark matter itself. Current null results from LHC searches for the supersymmetric partners of quarks typically exclude such particles with masses less than $\sim 1.5 \mathrm{TeV}$, although this limit is weakened in the case of mass-degenerate spectra.

A second mechanism produces the dark matter directly together with additional radiation from the initial quarks or gluons participating in the reaction, resulting in missing momentum recoiling against a single "mono-jet." Since this process does not rely as explicitly on the existence of additional colored particles that decay into dark matter, it is somewhat less sensitive to the details of the specific theory and places bounds directly in the parameter space of the dark matter mass and interaction strength. However, one does need to posit a specific form of the interaction between the dark matter and quarks or gluons. For electroweak-size couplings and specific choices of the interaction structure, these searches exclude dark matter masses below about $500 \mathrm{GeV}$.

High energy lepton colliders may create dark matter through analogous processes, such as production of dark matter along with a photon radiated from the initial leptons. For electroweaksize couplings of dark matter to electrons, LEP excluded dark matter masses below about $90 \mathrm{GeV}$. A future high-energy lepton collider could conceivably discover dark matter particles with masses up to roughly half the collision energy, e.g., $500 \mathrm{GeV}$ for a $1 \mathrm{TeV}$ ILC. For a list of current and proposed future colliders, see [50]. 


\section{Astrophysical Probes}

Non-gravitational interactions of dark matter affect a variety of astrophysical observables, including the number density and internal structure of galaxies. The majority of dark matter searches are focused on candidates that are astrophysically categorized as cold and collisionless dark matter (CDM). CDM predictions agree amazingly well with cosmological data [16], but the observed densities of dark matter in the central parts of galaxies are often lower than the simplest predictions.

Evidence for lowered central densities and constant central density cores is seen in the least massive to the most massive self-gravitating objects in the Universe, including satellite galaxies of the Milky Way, spiral galaxies, and clusters of galaxies. Constant density cores of dark matter are in conflict with the simplest CDM predictions, but baryonic feedback (such as outflows from supernovae) may change those predictions. Both feedback and deviations from CDM paradigm such as warm dark matter (WDM) or strongly self-interacting dark matter (SIDM) may be required to explain the data fully.

Compared to CDM, models of WDM have reduced power in small-scale density fluctuations and the number of low-mass dark matter halos is dramatically reduced. Hidden sector models where dark matter particles interact with other light particles can also lead to the same effects. These predictions can be tested in the future using strong gravitational lensing systems, precise observations of the clustering in the Universe and searches for new satellite galaxies and stellar streams. The mass-scale below which halo formation is suppressed is directly related to one or more parameters of the particle physics model - for example, mass and couplings of sterile neutrino dark matter candidates [49]. In addition, the central density of dark matter halos is also reduced in WDM cosmology, but large constant density cores have not been shown to form.

In comparison, the primary effect of SIDM is to reduce the central density of dark matter halos and create constant density (spherical) cores with observable effects if the cross section to particle mass ratio is in the $0.1-1 \mathrm{~cm}^{2} / \mathrm{g}$ range [51]. This range is consistent with measured dark matter halo shapes but larger cross sections are excluded by Bullet Cluster observations [10], and the predictions for this range may be tested with more sensitive observations of the Milky Way satellites at the faint end and clusters of galaxies at the bright end. Cross sections of this magnitude can be produced in hidden sector dark matter models through the exchange of a light gauge boson and this interaction can also endow the dark matter particle with the right relic density through a hidden sector analogue of the WIMP miracle [52].

In addition to structure formation, non-gravitational interactions of dark matter could impact a variety of other astrophysical phenomena. Coupling of axions and light sterile neutrinos (or generally any light hidden-sector particles) to standard model particles may affect the cooling of compact objects (stars, neutron stars, white dwarfs, supernovae) or the transparency of extragalactic background light to high-energy photons, which leads to stringent constraints on models [53. The observed fluctuations in the Cosmic Microwave Background (WMAP, Planck) are sensitive enough to the (small) dark matter annihilation rate during the era of recombination (age $\sim 380,000$ years) to be able to constrain thermal WIMP models [54. In the same vein, decays of dark matter particles (with lifetime much larger than the age of the Universe) can affect the reionization of neutral atoms about 13 billion years ago.

While dark matter physics may have imprinted tell-tale astrophysical signatures, it will be hard to unambiguously identify such signatures as non-gravitational interactions of dark matter. The complementarity with direct, indirect or collider searches is an essential part of this endeavor. 


\section{COMPLEMENTARITY}

\section{A. Qualitative Complementarity}

As evident from the brief descriptions in Sec. III, every experimental approach provides useful information for every dark matter scenario. At the same time, each approach is subject to different systematic uncertainties and no approach will illuminate all aspects of dark matter. In detail, what is learned from each approach is highly scenario-dependent.

At a qualitative level, the complementarity may be illustrated by the following observations that follow from basic features of each approach:

- Direct Detection is perhaps the most straightforward detection method, with excellent prospects for improved sensitivity in the coming decade and for discovering WIMPs. The approach requires careful control of low-energy backgrounds, and is relatively insensitive to dark matter that couples to leptons only, or to WIMP-like dark matter with mass $\sim 1 \mathrm{GeV}$ or below.

- Indirect Detection is sensitive to dark matter interactions with all standard model particles, directly probes the annihilation process suggested by the WIMP miracle, and experimental sensitivities are expected to improve greatly on several fronts in the coming decade. Discovery through indirect detection requires understanding astrophysical backgrounds and the signal strength is typically subject to uncertainties in halo profiles. Indirect detection signals are absent if dark matter annihilation is insignificant now, for example, as in the case of asymmetric dark matter.

- Particle Colliders provide the opportunity to study dark matter in a highly controlled laboratory environment, may be used to precisely constrain many dark matter particle properties, and are sensitive to the broad range of masses favored for WIMPs. Hadron colliders are relatively insensitive to dark matter that interacts only with leptons, and colliders are unable to distinguish missing momentum signals produced by a particle with lifetime $\sim 100 \mathrm{~ns}$ from one with lifetime $\gtrsim 10^{17} \mathrm{~s}$, as required for dark matter.

- Astrophysical Probes are unique probes of the "warmth" of dark matter and hidden dark matter properties, such as its self-interaction strength, and they measure the effects of dark matter properties on structure formation in the Universe. Astrophysical probes are typically unable to distinguish various forms of CDM from each other or make other precision measurements of the particle properties of dark matter.

\section{B. Quantitative Complementarity}

\section{Effective Operator Description}

The qualitative features outlined above may be illustrated in a simple and fairly modelindependent setting by considering dark matter that interacts with standard model particles through four-particle contact interactions, which represent the exchange of very heavy particles. These contact interactions are expected to work well to describe theories in which the exchanged particle mass is considerably larger than the momentum transfer of the physical process of interest.

To do this, we may choose representative, generation-independent, couplings of a spin- $1 / 2$ dark matter particle $\chi$ with quarks $q$, gluons $g$, and leptons $\ell$ (including neutrinos) given by

$$
\frac{1}{M_{q}^{2}} \bar{\chi} \gamma^{\mu} \gamma_{5} \chi \sum_{q} \bar{q} \gamma_{\mu} \gamma_{5} q+\frac{\alpha_{S}}{M_{g}^{3}} \bar{\chi} \chi G^{a \mu \nu} G_{\mu \nu}^{a}+\frac{1}{M_{\ell}^{2}} \bar{\chi} \gamma^{\mu} \chi \sum_{\ell} \bar{\ell} \gamma_{\mu} \ell .
$$



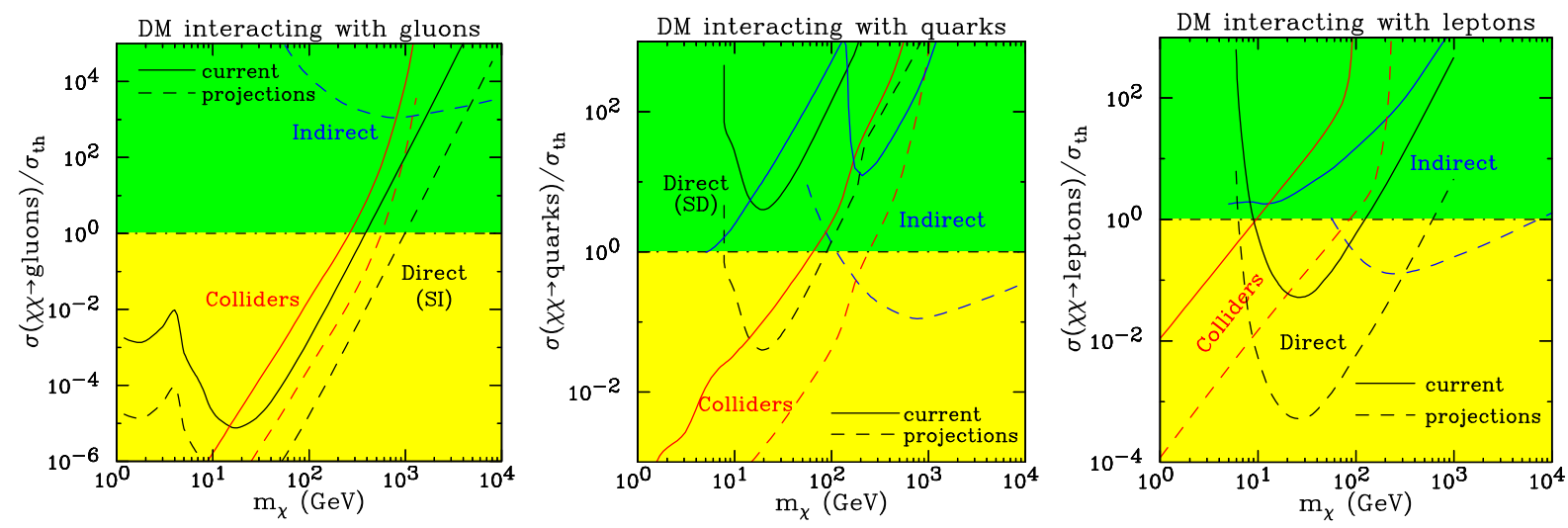

FIG. 2: Dark matter discovery prospects in the $\left(m_{\chi}, \sigma / \sigma_{\text {th }}\right)$ plane for current and future direct detection [55], indirect detection [56, 57], and particle colliders [58, 60] for dark matter coupling to gluons [61, quarks [61, 62, and leptons 63, 64, as indicated.

The interactions with quarks mediate spin-dependent direct signals, whereas those with gluons mediate spin-independent direct signals. The coefficients $M_{q}, M_{g}$, and $M_{\ell}$ characterize the strength of the interaction with the respective standard model particle, and in this representative example should be chosen such that the combined annihilation cross section into all three channels provides the correct relic density of dark matter. The values of the three interaction strengths, together with the mass of the dark matter particle $m_{\chi}$, completely define this theory and allow one to predict the rate of both spin-dependent and spin-independent direct scattering, the annihilation cross section into quarks, gluons, and leptons, and the production rate of dark matter at colliders.

Each class of dark matter search outlined in Sec. III is sensitive to some range of the interaction strengths for a given dark matter mass. Therefore, they are all implicitly putting a bound on the annihilation cross section into a particular channel. Since the annihilation cross section predicts the dark matter relic density, the reach of any experiment is thus equivalent to a fraction of the observed dark matter density. This connection can be seen in the plots in Fig. 2, which show the annihilation cross section normalized to the value $\sigma_{\text {th }}$, which is required ${ }^{2}$ for a thermal WIMP to account for all of the dark matter in the Universe. If the discovery potential for an experiment with respect to one of the interaction types reaches cross sections below $\sigma_{\text {th }}$ (the horizontal dot-dashed lines in Fig. 22, that experiment will be able to discover thermal relic dark matter that interacts only with that standard model particle and nothing else.

If an experiment were to observe an interaction consistent with an annihilation cross section below $\sigma_{\text {th }}$ (yellow-shaded regions in Fig. 2), it would have discovered dark matter but we would infer that the corresponding relic density is too large, and therefore there are important annihilation channels (or new physics that changes early Universe cosmology) still waiting to be discovered. Finally, if an experiment were to observe a cross section above $\sigma_{\text {th }}$ (green-shaded regions in Fig. 2), it would have discovered one species of dark matter, which, however, could not account for all of the dark matter (within this model framework), and consequently point to other dark matter species still waiting to be discovered.

In Fig. 2, we assemble the discovery potential and current bounds for several near-term dark matter searches that are sensitive to interactions with quarks and gluons, or leptons. It is clear that the searches are complementary to each other in terms of being sensitive to interactions with

\footnotetext{
${ }^{2}$ For non-thermal WIMPs, e.g. asymmetric DM, the annihilation cross-section does not have a naturally preferred value, but the plots in Fig. 2 are still meaningful.
} 
different standard model particles. These results also illustrate that within a given interaction type, the reach of different search strategies depends sensitively on the dark matter mass. For example, direct searches for dark matter are very powerful for masses around $100 \mathrm{GeV}$, but have difficulty at very low masses, where the dark matter particles carry too little momentum to noticeably affect heavy nuclei. This region of low mass is precisely where collider production of dark matter is easiest, since high energy collisions readily produce light dark matter particles with large momenta.

\section{Complete Models}

The effective theory description (1) of the dark matter interactions with standard model particles is an attempt to capture the salient features of the dark matter phenomenology without reference to any specific theoretical model. However, the complementarity between the different dark matter probes seen in Fig. 2 persists also when one considers specific well-motivated theoretical models. Among the many possible alternatives, low energy supersymmetry [65] has been the most popular and widely studied extension of the standard model, and we shall use it here as our second example. In supersymmetry, the DM candidate is generally the lightest neutralino $\tilde{\chi}_{1}^{0}$, which is its own antiparticle.

Even within the general framework of supersymmetry, there are many different model scenarios, distinguished by a number of input parameters $(\sim 20)$. A model-independent approach to supersymmetry is to scan over all those input parameters and consider all models that pass all existing experimental constraints and have a dark matter candidate which could explain at least a portion of the observed dark matter density [66]. Results from such model-independent scans with over 200,000 points are shown in Fig. 3, where each dot represents one particular supersymmetric model. Within each model, the dark matter interactions are completely specified and one can readily compute all relevant dark matter signals. The models are categorized depending on the observability of a dark matter signal in direct detection experiments (green points), indirect detection experiments (blue points) or both (red points). The gray points represent models that escape detection in dark matter experiments, but may be discovered at the upgraded LHC, if the mass of the lightest colored superpartner is within $m_{\text {LCSP }} \sim 3 \mathrm{TeV}$. A sizable fraction of models (the blue points) can only be seen in indirect detection (via ground-based gamma ray telescopes). Another large fraction of models (the gray points) can only be seen at the LHC. Figure 3 demonstrates that the three different dark matter probes nicely combine to discover many (albeit not all) supersymmetry models in this scan.

\section{Post-Discovery Complementarity}

As important as a broad program of complementary searches is to establishing a compelling signal for dark matter, it becomes even more important after a signal has been reported for several reasons.

First, as is well known, many tentative dark matter signals have already been reported. The potential identification of a quarter of the Universe will require extraordinary proof in the form of verification by other experiments.

Second, each search strategy has its limitations. For example, as noted in Sec. IVA, the discovery of a dark matter signal at particle colliders only establishes the production of a particle with lifetime greater than about $100 \mathrm{~ns}$. The assumption that this particle contributes to dark matter requires an extrapolation in lifetime of 24 orders of magnitude! It is only by corroborating a particle collider discovery through another method that one can claim that the collider discovery is relevant for cosmology. 

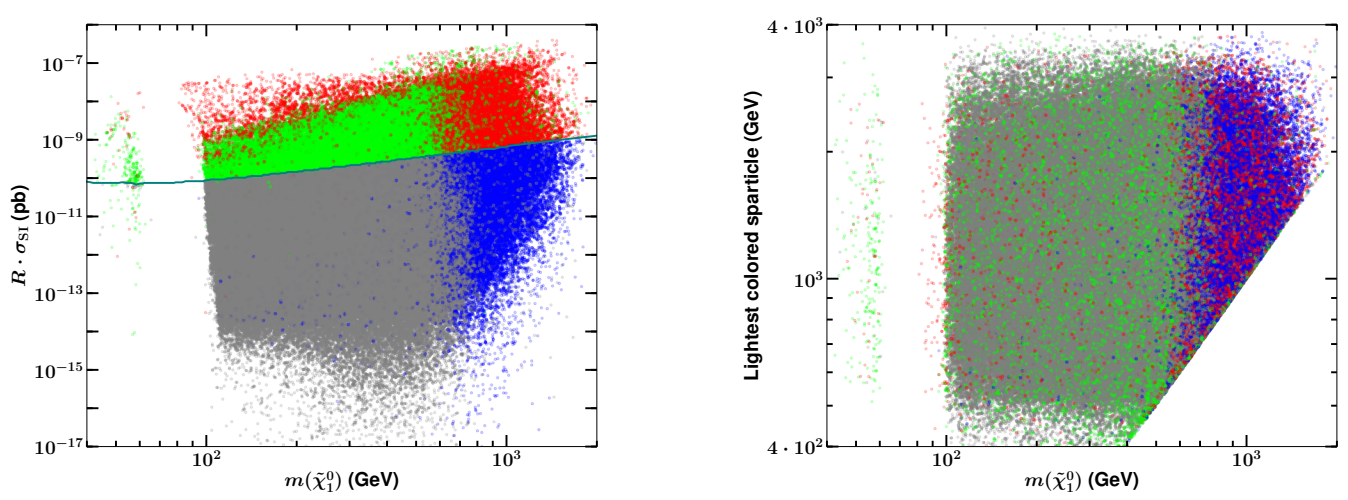

FIG. 3: Results from a model-independent scan 66, 67 of the full parameter space in the minimal supersymmetric model (MSSM), presented in the $\left(m_{\chi}, R \cdot \sigma_{\mathrm{SI}}^{p}\right)$ plane (left panel) or the $\left(m_{\chi}, m_{\mathrm{LCSP}}\right)$ plane (right panel), where $R=\Omega_{\chi} / \Omega_{\mathrm{DM}}$. The models are divided into categories, depending on whether dark matter can be discovered in future direct detection experiments (green points), indirect detection experiments (blue points) or both (red points). The gray points represent models that may be discovered at the upgraded LHC, but escape detection in future direct or indirect detection experiments.

Last, the discovery of dark matter will usher in a rich and decades-long program of dark matter studies. Consider the following (somewhat optimistic) scenario: The LHC sees a missing energy signal, and precision measurements find evidence that it is due to a $60 \mathrm{GeV}$ neutralino. This result is confirmed by direct search experiments, which discover a signal consistent with this mass. However, further LHC and ILC studies constrain the neutralino's predicted thermal relic density $\Omega_{\chi}$ to be half of $\Omega_{\mathrm{DM}}$, implying that it is not a thermal relic, or that it makes up only half of the dark matter. The puzzle is resolved when axion detectors discover a signal, which is consistent with axions making up the rest of the dark matter, and progress in astrophysical theory, simulations, and observations leads to a consistent picture with dark matter composed entirely of CDM. The combined data establish a new standard cosmology in which dark matter is composed of equal parts neutralinos and axions, and extend our understanding of the early Universe back to neutralino freezeout, just 1 ns after the Big Bang. Direct and indirect detection rates are then used to constrain the local dark matter density, halo profiles, and substructure, establishing the new fields of neutralino and axion astronomy.

This two-component scenario is more complicated than assumed in many dark matter studies, but it is still relatively simple - as is often noted, the visible Universe has many components, and there is no reason that the dark Universe should be any simpler. As simple as this scenario is, however, it illustrates the point that, even for dark matter candidates that we have studied and understood, the information provided by several approaches will be essential to understanding the particle nature of dark matter and its role in astrophysics and cosmology. A balanced program with components in each of the four approaches is required to cover the many well-motivated dark matter possibilities, and their interplay will likely be essential to realize the full potential of upcoming discoveries.

\section{CONCLUSIONS}

The problem of identifying dark matter is central to the fields of particle physics and astrophysics, and has become a leading problem in all of basic science. In the coming decade, the field of dark matter will be transformed, with a perfect storm of experimental and technological progress 
set to put the most promising ideas to the test.

Dark matter searches rely on four approaches or pillars: direct detection, indirect detection, particle colliders, and astrophysical probes. In this Report, we have described the complementary relation of these approaches to each other. This complementarity may be seen on several levels. First, these approaches are qualitatively complementary: they differ in essential characteristics, and they rely on different dark matter properties to see a signal. A complementary set of approaches is required to be sensitive to the dark matter possibilities that are currently both viable and wellmotivated. The approaches are also quantitatively complementary: within a given class of dark matter possibilities, these approaches are sensitive to different dark matter interactions and mass ranges.

Last, the discovery of a compelling dark matter signal is only the beginning. Complementary experiments are required to verify the initial discovery, to determine whether the particle makes up all of dark matter or only a portion, and to identify its essential properties, such as its interactions, spin, and mass, and to determine its role in forming the large scale structures of the Universe that we see today. A balanced dark matter program is required to carry out this research program to discover and study dark matter and to transform our understanding of the Universe on both the smallest and largest length scales.

[1] S. Arrenberg, H. Baer, V. Barger, L. Baudis, D. Bauer, et al., "Working Group Report: Dark Matter Complementarity," arXiv:1310.8621 [hep-ph].

[2] F. Zwicky, "Spectral displacement of extra galactic nebulae," Helv. Phys. Acta 6 (1933) 110-127.

[3] V. C. Rubin and W. K. Ford, Jr., "Rotation of the Andromeda Nebula from a Spectroscopic Survey of Emission Regions," Astrophys. J. 159 (1970) 379-403.

[4] V. C. Rubin, N. Thonnard, and W. K. Ford, Jr., "Rotational properties of 21 SC galaxies with a large range of luminosities and radii, from NGC $4605(\mathrm{R}=4 \mathrm{kpc})$ to UGC 2885 (R=122 kpc)," Astrophys. J. 238 (1980) 471.

[5] A. Bosma, The distribution and kinematics of neutral hydrogen in spiral galaxies of various morphological types. PhD thesis, PhD Thesis, Groningen Univ., (1978), 1978.

[6] A. Refregier, "Weak Gravitational Lensing by Large-Scale Structure," Ann. Rev. Astron. Astrophys. 41 (2003) 645-668, arXiv: astro-ph/0307212.

[7] J. A. Tyson, G. P. Kochanski, and I. P. Dell'Antonio, "Detailed Mass Map of CL0024+1654 from Strong Lensing," Astrophys. J. 498 (1998) L107, arXiv:astro-ph/9801193.

[8] A. D. Lewis, D. A. Buote, and J. T. Stocke, "Chandra Observations of Abell 2029: The Dark Matter Profile at $<0.01 R_{\text {vir }}$ in an Unusually Relaxed Cluster," Astrophys. J. 586 (2003) 135-142, arXiv: astro-ph/0209205.

[9] S. W. Allen, A. C. Fabian, R. W. Schmidt, and H. Ebeling, "Cosmological constraints from the local X-ray luminosity function of the most X-ray luminous galaxy clusters," Mon. Not. Roy. Astron. Soc. 342 (2003) 287, arXiv: astro-ph/0208394.

[10] D. Clowe et al., "A direct empirical proof of the existence of dark matter," Astrophys. J. 648 (2006) L109-L113, arXiv:astro-ph/0608407.

[11] Particle Data Group Collaboration, B. D. Fields and S. Sarkar, "Big Bang nucleosynthesis," Phys. Lett. B667 (2008) 228-231.

[12] Supernova Search Team Collaboration, A. G. Riess et al., "Observational Evidence from Supernovae for an Accelerating Universe and a Cosmological Constant," Astron. J. 116 (1998) 1009-1038, arXiv:astro-ph/9805201.

[13] Supernova Cosmology Project Collaboration, S. Perlmutter et al., "Measurements of Omega and Lambda from 42 High-Redshift Supernovae," Astrophys. J. 517 (1999) 565-586, arXiv: astro-ph/9812133.

[14] SDSS Collaboration, M. Tegmark et al., "Cosmological parameters from SDSS and WMAP," Phys.Rev. D69 (2004) 103501, arXiv:astro-ph/0310723 [astro-ph]. 
[15] E. Hawkins, S. Maddox, S. Cole, D. Madgwick, P. Norberg, et al., "The 2dF Galaxy Redshift Survey: Correlation functions, peculiar velocities and the matter density of the universe," Mon.Not.Roy.Astron.Soc. 346 (2003) 78, arXiv:astro-ph/0212375 [astro-ph].

[16] E. Komatsu et al., "Seven-Year Wilkinson Microwave Anisotropy Probe (WMAP) Observations: Cosmological Interpretation," arXiv:1001.4538 [astro-ph.CO].

[17] Planck Collaboration, P. Ade et al., "Planck 2013 results. XVI. Cosmological parameters," arXiv:1303.5076 [astro-ph.CO].

[18] G. Bertone, ed., Particle Dark Matter: Observations, Models and Searches. Cambridge University Press, 2010.

[19] L. Bergstrom, "Dark Matter Candidates," New J.Phys. 11 (2009) 105006, arXiv:0903.4849 [hep-ph].

[20] J. L. Feng, "Dark Matter Candidates from Particle Physics and Methods of Detection," Ann.Rev.Astron.Astrophys. 48 (2010) 495-545, arXiv:1003.0904 [astro-ph.C0].

[21] Y. B. Zeldovich Adv. Astron. Astrophys. 3 (1965) 241.

[22] H.-Y. Chiu, "Symmetry between particle and anti-particle populations in the universe," Phys. Rev. Lett. 17 (1966) 712 .

[23] G. Steigman, "Cosmology Confronts Particle Physics," Ann. Rev. Nucl. Part. Sci. 29 (1979) 313-338

[24] R. J. Scherrer and M. S. Turner, "On the Relic, Cosmic Abundance of Stable Weakly Interacting Massive Particles," Phys. Rev. D33 (1986) 1585.

[25] H. Goldberg, "Constraint on the Photino Mass from Cosmology," Phys.Rev.Lett. 50 (1983) 1419.

[26] J. R. Ellis, J. Hagelin, D. V. Nanopoulos, K. A. Olive, and M. Srednicki, "Supersymmetric Relics from the Big Bang," Nucl.Phys. B238 (1984) 453-476.

[27] G. Servant and T. M. Tait, "Is the lightest Kaluza-Klein particle a viable dark matter candidate?," Nucl.Phys. B650 (2003) 391-419, arXiv:hep-ph/0206071 [hep-ph].

[28] H.-C. Cheng, J. L. Feng, and K. T. Matchev, "Kaluza-Klein dark matter," Phys.Rev.Lett. 89 (2002) 211301, arXiv:hep-ph/0207125 [hep-ph].

[29] S. Nussinov, "Technocosmology: Could a Technibaryon Excess Provide a 'Natural' Missing Mass Candidate?," Phys.Lett. B165 (1985) 55.

[30] D. B. Kaplan, "A Single explanation for both the baryon and dark matter densities," Phys.Rev.Lett. 68 (1992) 741-743.

[31] S. M. Barr, "Baryogenesis, sphalerons and the cogeneration of dark matter," Phys.Rev. D44 (1991) 3062-3066.

[32] D. E. Kaplan, M. A. Luty, and K. M. Zurek, "Asymmetric Dark Matter," Phys.Rev. D79 (2009) 115016, arXiv:0901.4117 [hep-ph]

[33] A. Kusenko and L. J. Rosenberg, "Working Group Report: Non-WIMP Dark Matter," arXiv:1310.8642 [hep-ph].

[34] R. D. Peccei and H. R. Quinn, "Constraints Imposed by CP Conservation in the Presence of Instantons," Phys. Rev. D16 (1977) 1791-1797.

[35] S. Weinberg, "A New Light Boson?," Phys. Rev. Lett. 40 (1978) 223-226.

[36] F. Wilczek, "Problem of Strong p and t Invariance in the Presence of Instantons," Phys. Rev. Lett. 40 (1978) 279-282.

[37] S. J. Asztalos, L. J. Rosenberg, K. van Bibber, P. Sikivie, and K. Zioutas, "Searches for astrophysical and cosmological axions," Ann.Rev.Nucl.Part.Sci. 56 (2006) 293-326.

[38] S. Dodelson and L. M. Widrow, "Sterile Neutrinos as Dark Matter," Phys. Rev. Lett. 72 (1994) 17-20, arXiv:hep-ph/9303287

[39] A. Kusenko, "Sterile neutrinos: the dark side of the light fermions," Phys. Rept. 481 (2009) 1-28, arXiv:0906.2968 [hep-ph].

[40] K. Abazajian, M. Acero, S. Agarwalla, A. Aguilar-Arevalo, C. Albright, et al., "Light Sterile Neutrinos: A White Paper," arXiv:1204.5379 [hep-ph].

[41] M. W. Goodman and E. Witten, "Detectability of Certain Dark Matter Candidates," Phys.Rev. D31 (1985) 3059 .

[42] P. Cushman, C. Galbiati, D. McKinsey, H. Robertson, T. Tait, et al., "Working Group Report: WIMP Dark Matter Direct Detection," arXiv:1310.8327 [hep-ex].

[43] "Table of Planned and Current Experiments." http://www . snowmass2013.org/tiki-index .php?page=SLAC 
[44] P. Sikivie, "Experimental Tests of the Invisible Axion," Phys.Rev.Lett. 51 (1983) 1415.

[45] J. Buckley, D. Cowen, S. Profumo, A. Archer, M. Cahill-Rowley, et al., "Working Group Report: WIMP Dark Matter Indirect Detection," arXiv:1310.7040 [astro-ph.HE].

[46] "Indirect Detection Experiments." http://www . snowmass2013.org/tiki-index.php?page=WIMP+Dark+Matter+Indirect+Detection

[47] A. Geringer-Sameth and S. M. Koushiappas, "Exclusion of canonical WIMPs by the joint analysis of Milky Way dwarfs with Fermi," Phys.Rev.Lett. 107 (2011) 241303, arXiv:1108.2914 [astro-ph.CO]

[48] K. Abazajian, G. M. Fuller, and W. H. Tucker, "Direct detection of warm dark matter in the X-ray," Astrophys.J. 562 (2001) 593-604, arXiv:astro-ph/0106002 [astro-ph].

[49] A. Kusenko, "Sterile neutrinos, dark matter, and the pulsar velocities in models with a Higgs singlet," Phys. Rev. Lett. 97 (2006) 241301, arXiv:hep-ph/0609081.

[50] "Energy Frontier Facilities List." http://www . snowmass2013.org/tiki-index .php?page=EF+Facilities+List.

[51] D. N. Spergel and P. J. Steinhardt, "Observational evidence for selfinteracting cold dark matter," Phys.Rev.Lett. 84 (2000) 3760-3763, arXiv: astro-ph/9909386 [astro-ph].

[52] J. L. Feng, M. Kaplinghat, and H.-B. Yu, "Halo Shape and Relic Density Exclusions of Sommerfeld-Enhanced Dark Matter Explanations of Cosmic Ray Excesses," Phys.Rev.Lett. 104 (2010) 151301, arXiv:0911.0422 [hep-ph].

[53] G. Raffelt, "Astrophysics probes of particle physics," Phys.Rept. 333 (2000) 593-618.

[54] N. Padmanabhan and D. P. Finkbeiner, "Detecting dark matter annihilation with CMB polarization: Signatures and experimental prospects," Phys.Rev. D72 (2005) 023508, arXiv: astro-ph/0503486 [astro-ph].

[55] "The Dark Matter Community Website." http://dmtools.brown.edu/.

[56] Fermi-LAT Collaboration, M. Ackermann et al., "Constraining Dark Matter Models from a Combined Analysis of Milky Way Satellites with the Fermi Large Area Telescope," Phys.Rev.Lett. 107 (2011) 241302, arXiv:1108.3546 [astro-ph.HE].

[57] M. Wood, J. Buckley, S. Digel, S. Funk, D. Nieto, M. Sanchez-Conde, et al., "CF2 White Paper: Prospects for Indirect Detection of Dark Matter with CTA." http://www.snowmass2013.org/tiki-download_file.php?fileId=68.

[58] CMS Collaboration, S. Chatrchyan et al., "Search for dark matter and large extra dimensions in monojet events in $p p$ collisions at $\sqrt{s}=7 \mathrm{TeV}$," JHEP 1209 (2012) 094, arXiv:1206.5663 [hep-ex].

[59] ATLAS Collaboration, G. Aad et al., "Search for dark matter candidates and large extra dimensions in events with a jet and missing transverse momentum with the ATLAS detector," arXiv:1210.4491 [hep-ex].

[60] L. Vacavant and I. Hinchliffe, "Signals of models with large extra dimensions in ATLAS," J.Phys. G27 (2001) 1839-1850.

[61] J. Goodman, M. Ibe, A. Rajaraman, W. Shepherd, T. M. Tait, et al., "Constraints on Dark Matter from Colliders," Phys.Rev. D82 (2010) 116010, arXiv:1008.1783 [hep-ph].

[62] M. Beltran, D. Hooper, E. W. Kolb, and Z. C. Krusberg, "Deducing the nature of dark matter from direct and indirect detection experiments in the absence of collider signatures of new physics," Phys.Rev. D80 (2009) 043509, arXiv:0808.3384 [hep-ph].

[63] P. J. Fox, R. Harnik, J. Kopp, and Y. Tsai, "LEP Shines Light on Dark Matter," Phys.Rev. D84 (2011) 014028, arXiv:1103.0240 [hep-ph].

[64] Y. J. Chae and M. Perelstein, "Dark Matter Search at a Linear Collider: Effective Operator Approach," arXiv:1211.4008 [hep-ph].

[65] S. P. Martin, "A Supersymmetry primer," arXiv:hep-ph/9709356 [hep-ph].

[66] M. W. Cahill-Rowley, J. L. Hewett, S. Hoeche, A. Ismail, and T. G. Rizzo, "The New Look pMSSM with Neutralino and Gravitino LSPs," Eur.Phys.J. C72 (2012) 2156, arXiv:1206.4321 [hep-ph].

[67] M. Cahill-Rowley, R. Cotta, A. Drlica-Wagner, S. Funk, J. Hewett, A. Ismail, T. Rizzo, and M. Wood, "Complementarity and Searches for Dark Matter in the pMSSM." white paper contributed to Snowmass 2013. 